\title{
Une mutation de la protéine Gs $\alpha$ responsable d'hyper- et d'hypo-fonctionnements endocrines
}

Une mutation génétique peut-elle être à la fois "activatrice " et «inhibitrice" ? Le bon sens ferait répondre non. C'est pourtant le cas d'une mutation de Gs, membre célèbre de la famille des protéines $\mathrm{G}$ hétérotrimériques $(\alpha \beta \gamma)$, qui vient d'être honorée par le prix Nobel de Médecine (m/s $n^{\circ} 11$, vol. 10, p.1183). Gs permet de coupler des récepteurs membranaires à l'enzyme adénylyl cyclase, qui élève le taux intracellulaire d'AMPc. La mutation en question concerne sa sous-unité $\alpha$ (Gs $\alpha)$, dans laquelle l'alanine en position 366 est remplacée par une sérine (A366S). Cette mutation est retrouvée chez des enfants qui associent deux maladies génétiques endocriniennes: pseudohypoparathyroïdie de type IA et testotoxicose. Le paradoxe est que ces maladies sont toutes deux associées à une modification de l'activité de Gs, mais de sens opposé : déficit pour la pseudohypoparathyroïdie, augmentation pour la testotoxicose. L'explication moléculaire de ce paradoxe vient d'être apportée par le groupe de Henri Bourne $\left(\mathrm{m} / \mathrm{s} n^{\circ} 12\right.$, vol. 10, p. 1177, [1, 2]). Elle est, bien sûr, très élégante.

Les maladies associées à cette mutation A366S ont les caractéristiques suivantes : (1) la pseudohypoparathyroïdie de type $1 \mathrm{~A}$ associe un syndrome dysmorphique (ostéodystrophie d'Albright) et une résistance aux hormones qui élèvent le taux d'AMPc intracellulaire $\left(m / s \quad n^{\circ} 2\right.$, vol. $8, p .184)$, [3]. La résistance à la PTH (hormone parathyroïdienne hypercalcémiante) est la plus marquée, elle réalise une hypocalcémie avec taux élevé de PTH à laquelle la maladie doit son nom. Les patients présentent également une résistance aux $\mathrm{m} / \mathrm{s} n^{\circ} \mathrm{l}$, vol. 11 , janvier 95 hormones thyréostimulante (TSH), folliculostimulante (FSH), lutéotrope (I.H), et au glucagon. Ces résistances hormonales multiples sont en rapport avec une baisse généralisée de 50) \% de l'activité de Gs, qu'il est facile de mesurer in vitro au niveau érythrocytaire. Ce déficit traduit la perte de fonction d'un allèle de Gs $\alpha$, du fait d'une mutation hétérozygote inactivatrice. I.es mutations identifiées jusqu'ici affectent le démarrage de la transcription du gène de Gs $\alpha$ ou l'épissage de son transcrit. Elles ont pour conséquence l'abolition de l'expression de l'allèle muté [4], ou une délétion de l'extrémité $\mathrm{N}$-terminale de la protéine, qui est impliquée dans le couplage de Gs $\alpha$ au récepteur hormonal [5]. (2) La testotoxicose se manif este par l'apparition de signes de puberté vers l'âge de 4 ans chez le garçon. Il s'agit d'une pseudo-puberté précoce, en rapport avec une hypersécrétion de testostérone d'origine primitivement testiculaire. On rappelle que la testostérone est sécrétée par les cellules de I.eydig du testicule, qui sont normalement sous le contrôle de l'hormone hypophysaire I.H. I.a I.H agit sur un récepteur testiculaire couplé à Gs pour stimuler la sécrétion de testostérone ; en retour, la testostérone agit sur les cellules hypophysaires gonadotropes pour freiner la sécrétion de I.H. Dans la testotoxicose, cette classique boucle de rétrocontrôle est inefficace, car l'hypersécrétion de testostérone persiste malgré le freinage de la sécrétion de I.H : les cellules de I.eydig sont devenues "autonomes". Une explication moléculaire de cette autonomie de sécrétion a été récemment fournie par la mise en évidence d'une mutation activatrice du récep- teur de la L.H chez des patients atteints de testotoxicose familiale [6]. En effet, le récepteur muté a la propriété d'activer Gs et l'adénylyl cyclase même en l'absence de L.H, ce qui permet aux cellules de Leydig d'échapper au contrôle hypophysaire.

La pseudohypoparathyroïdie de type 1A traduit donc un déficit généralisé de Gs, et la testotoxicose une activation de Gs dans le testicule. Comment expliquer qu'une même mutation de $\mathrm{Gs} \alpha$ donne naissance à ces maladies apparemment opposées? Pour répondre à cette question, Henri Bourne el al. ont produit et purifié les protéines Gs $\alpha$ mutée et normale en utilisant le système d'expression de baculovirus dans les cellules d'insecte. Ils ont pu alors étudier les propriétés biochimiques de la protéine mutée, et les comparer à celles de la protéine normale.

Dans un premier temps, la protéine mutante fut incubée avec des membranes de cellules déficientes en Gs (les fameuses cyc-, qui ont permis d'isoler (ss). Les conditions expérimentales sont importantes à préciser: l'incubation était faite à température ambiante, $20{ }^{\circ} \mathrm{C}$, et en présence de GTP. Ils constatèrent alors que la protéine mutante entraînait une activation de l'adénylyl cyclase bien supérieure à la protéine normale. Dans ces conditions expérimentales, la mutation était donc bien activatrice, et Bourne el al. recherchèrent le mécanisme de cette activation.

Il faut rappeler ici que l'activité des protéines $G$ dépend de leur liaison au GDP ou au GTP, qui se fait sur la sous-unité $\alpha$. L.es protéines G fonctionment en effet selon un cycle entre 


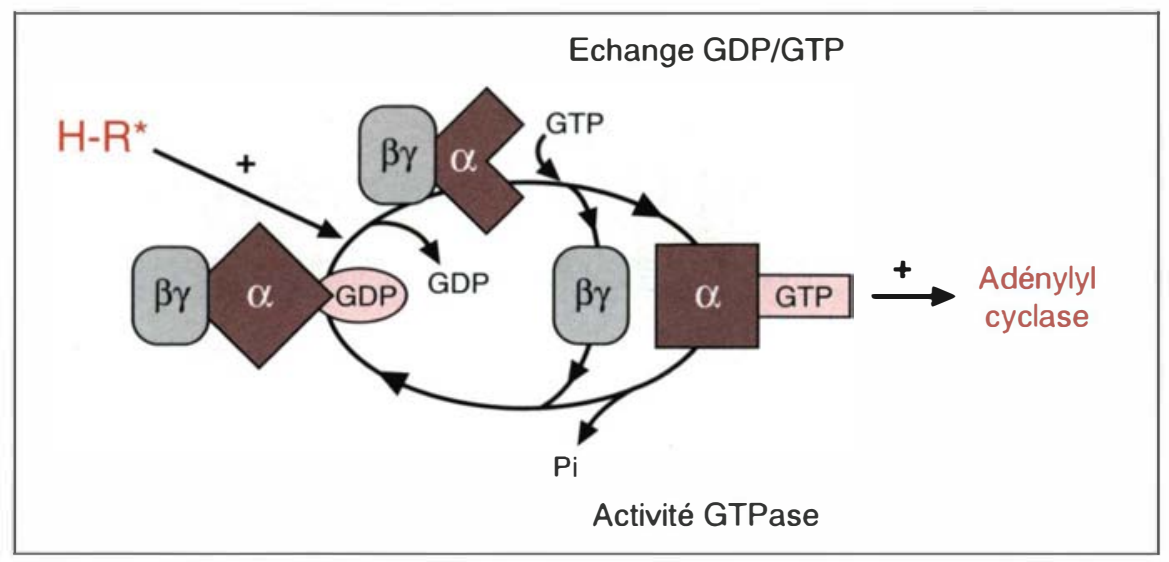

Figure 1. Cycle d'activation/inactivation des protéines $G$. Les protéines $G$ fonctionnent selon un cycle entre un état inactif, où $\alpha$ est associée à $\beta \gamma$ et lie du GDP, et un état actif, dans lequel $\alpha$ lie du GTP ( $\alpha-G T P)$ et s'est dissociée de $\beta \gamma$. L'activation dépend de l'échange GDP/GTP, qui est très faible en l'absence de récepteur activé. Lorsque le récepteur est stimulé par une hormone ou un agoniste $\left(R-H^{*}\right)$, il accélère la constante de dissociation du GDP de $\alpha$, qui peut lier un GTP en échange. Au cours de cet échange, le complexe $\alpha \beta \gamma$ passe par un état "vide" avant de lier le GTP et se dissocier en $\alpha$-GTP et $\beta \gamma$. L'inactivation dépend de l'hydrolyse du GTP en GDP par une activité GTPase intrinsèque à $\alpha$, (mais qui peut être influencée par $d^{\prime}$ autres protéines). $\alpha$ - GDP se réassocie alors à $\beta \gamma$.

un état inactif, dans lequel la sousunité $\alpha$ lie du GDP ( $\alpha$-GDP) et est associée aux sous-unités $\beta \gamma$, et un état actif, dans lequel $\alpha$ lie du GTP $(\alpha$ GTP) et s'est dissociée de $\beta \gamma$ (figure 1). Le passage de l'état inactif à l'état actif nécessite que le GDP se dissocie de $\alpha$, qui passe par un état "vide» avant de lier un GTP en échange. Le retour à l'état inactif est assuré par l'hydrolyse du GTP en GDP, qui dépend d'une activité GTPase intrinsèque à $\alpha$. En l'absence de récepteur hormonal activé, l'équilibre du système est en faveur de la forme inactive $\alpha$-GDP. En effet, la constante cinétique de dissociation du GDP de $\alpha$ est alors très faible, et l'activité d'échange GDP/GTP de $\alpha$ est négligeable par rapport à l'activité GTPase. En revanche, en présence d'agoniste, le récepteur acquiert la capacité d'augmenter la constante cinétique de dissociation du GDP de $\alpha$, et donc la fréquence de dissociation du GDP. L'échange GDP/GTP augmente, et l'hydrolyse du GTP devient
80 fois plus vite que la protéine native. L'augmentation de l'hydrolyse du GTP n'était donc que la conséquence d'une augmentation bien plus forte de l'échange GDP/GTP, rendant l'hydrolyse du GTP limitante : le résultat était une augmentation du taux de $\alpha$-GTP et donc une activation de l'adénylyl cyclase (figure 2). I.a conclusion est donc clairement que A366S active Gs de la même façon qu'un récepteur hormonal, en augmentant la vitesse de dissociation du GDP. Il est ainsi possible d'expliquer la testotoxicose des patients porteurs de A366S par un mécanisme très similaire à celui décrit chez des patients porteurs d'une mutation activatrice du récepteur de la $\mathrm{LH}$.

Fort bien, mais comment expliquer alors la résistance à la $\mathrm{PTH}$, à la TSH? La clé de l'histoire se trouve dans l'environnement, qui a toujours sa place, même dans les maladies génétiques. En effet, l'environnement du testicule n'est pas le même que celui du reste du corps : la température y est plus fraîche de 2 à $5^{\circ} \mathrm{C}$ ! Bourne et al. étudièrent alors logiquement l'effet de la température sur la stabilité de A366S, et ils trouvèrent qu'à $37^{\circ} \mathrm{C}$ la demi-vie de la protéine mutée était inférieure à une heure, contre 21 heures pour la protéine native. Ainsi le phénotype pseudohypoparathyroïdie de type $1 \mathrm{~A}$ s'expliquait par la thermosensibilité de la protéine mutante, qui annihilait l'effet activateur de la mutation dans toutes les régions du corps à $37^{\circ} \mathrm{C}$.

CQFD, mais pourquoi la protéine mutante est-elle plus rapidement dénaturée que la protéine sauvage? Là réside l'élégance de la démonstration : l'augmentation de la vitesse de dissociation du GDP de la protéine mutante explique à la fois son activation et sa thermosensibilité. En effet, Bourne et al. démontrent à propos de Gs un fait déjà établi à propos d'autres protéines $G$ comme la transducine, à savoir que le taux de dénaturation est proportionnel à la fréquence de l'état "vide» (on rappelle que cet état vide est l'état intermédiaire dans lequel $\alpha$ a relâché un GDP mais n'a pas encore lié un GTP en échange). Comme A366S échange son GDP plus fréquemment que Gs sauvage, elle se retrouve plus souvent ve depl hypophysaires ou thy miment l'effet de la GHRH ou de la $\mathrm{TSH}\left(\mathrm{m} / \mathrm{s} n^{\circ} 12\right.$, vol. 9, p. 1421). Bourne et al. ont donc mesuré l'activité GTPase de A366S : celle-ci n'est pas inhibée, mais au contraire activée (d'un facteur 20). Les chercheurs se sont alors tournés vers l'échange GDP/GTP et ont observé que la protéine mutante échangeait son GDP 
dans l'état vide, dénaturable. Or la dénaturation est un phénomène thermodynamique qui s'accélère lorsque la température augmente. La température devient ainsi déterminante pour l'activité de la protéine : à $32^{\circ} \mathrm{C}$ la balance penche vers l'activation, mais à $37^{\circ} \mathrm{C}$ vers la dénaturation (figure 2).

Comme toujours, la découverte d'une mutation pathologique offre des perspectives à la fois aux cliniciens et aux fondamentalistes. Pour ces derniers, l'effet de la mutation A 3665 pose le problème des relations structure/fonction de la région de Gs qui contient l'alanine 366. Cette région présente une séquence très conservée, G5, dont la structure tridimensionnelle a été déterminée dans le cas de la transducine : elle constitue une boucle dont les résidus stabilisent le noyau guanine du GDP et GTP. Le remplacement de l'alanine 366 par une sérine entraîne probablement une déstabilisation du noyau guanine, à l'origine de la dissociation accélérée du GDP. L'intérêt de ce modèle est qu'il permet d'émettre l'hypothèse que l'activation des protéines $G$ par le récepteur fait également intervenir la région G5, par un mécanisme qui reste à préciser.

Pour les cliniciens, les résultats de Bourne et al. permettent plusieurs prédictions. Tout d'abord, comme le suggère Bourne lui-même, un moyen simple de traiter la testotoxicose des garçons porteurs de la mutation serait de ramener leurs testicules à $37^{\circ} \mathrm{C}$. En fait, il existe une situation où les testicules sont déjà à $37^{\circ} \mathrm{C}$ : la cryptorchidie, anomalie qui se caractérise par des testicules en position abdominale du fait d'un trouble de leur migration. Le retentissement de la cryptorchidie sur le testicule est triple : non seulement elle provoque une insuffisance endocrine (déficit de sécrétion de testostérone), mais également une insuffisance exocrine (déficit de la spermatogenèse) et un risque élevé de cancérisation. Cela suggère que les effets de l'élévation de la température testiculaire ne se limitent pas à Gs, mais affectent d'autres molécules, sans doute par le biais d'une modification de leur activité enzymatique ou de leur taux de $\mathrm{m} / \mathrm{s} n^{\circ}$ l, vol. II, janvier 95

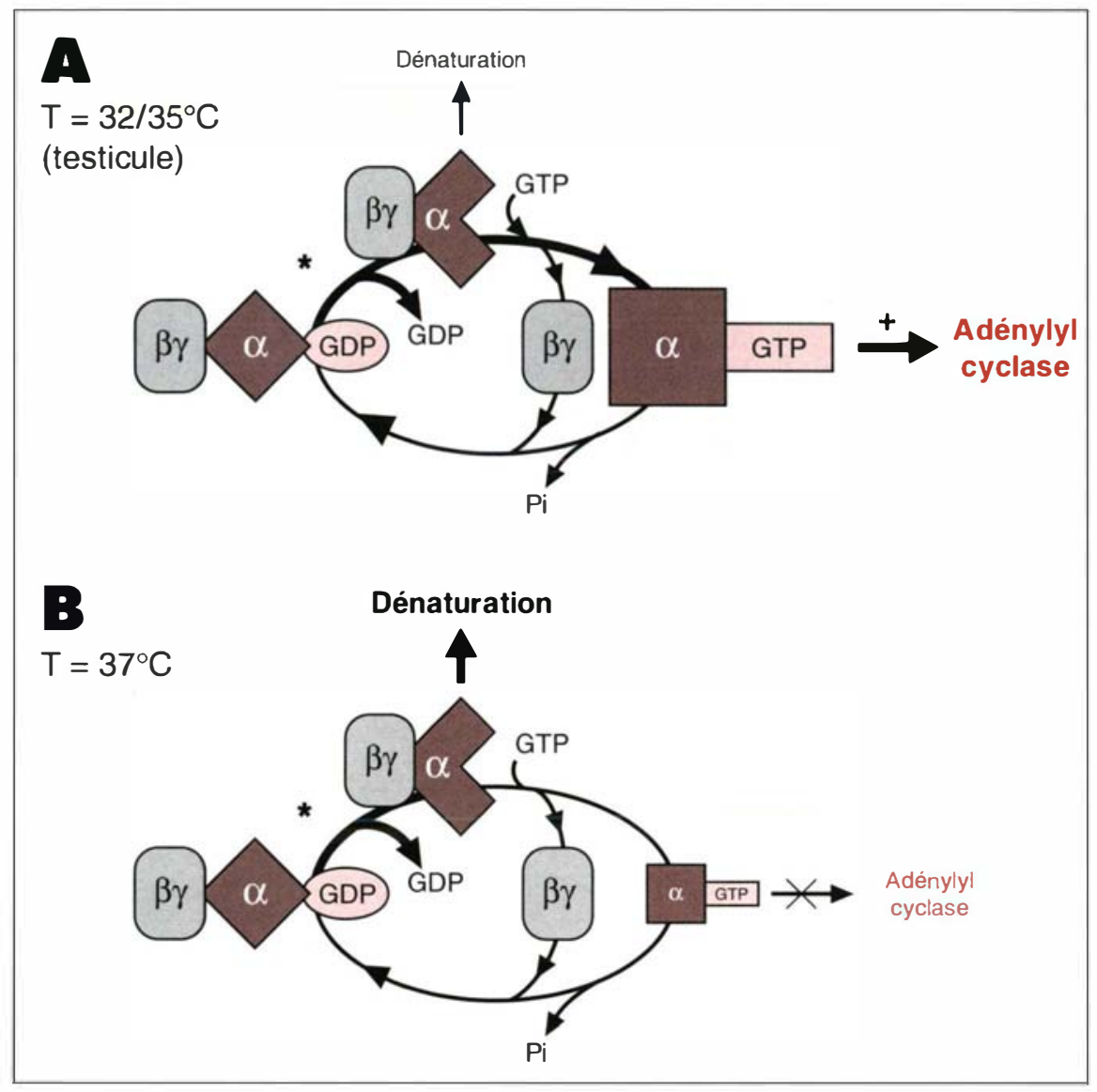

Figure 2. Effets opposés de la mutation A366S de Gso à $32^{\circ} \mathrm{C}$ et à $37^{\circ} \mathrm{C}$. La protéine mutée a une activité d'échange GDP/GTP spontanément très élevée, car la constante cinétique de dissociation du GDP est élevée même en l'absence de récepteur activé. Cela augmente la fréquence de l'état vide, qui est une forme dénaturable. A. A $32^{\circ} \mathrm{C}$, la dénaturation reste faible et l'équilibre se trouve déplacé vers la forme active $\alpha$-GTP, car l'activité GTPase devient limitante. II y a donc activation de l'adénylyl cyclase indépendamment de la présence d'hormone. B. A $37^{\circ} \mathrm{C}$, la forme vide est rapidement dénaturée, le taux de $\alpha$-GTP diminue et ne peut être stimulé. II y a résistance à l'action hormonale.

dénaturation. Deuxièmement, il est probable que de nouveaux phénotypes liés à la mutation A366S seront bientôt découverts, ou redécouverts. Tout d'abord, dans le sexe féminin, où les ovaires sont à l'intérieur du corps et donc à $37^{\circ} \mathrm{C}$. Chez la fille, le phénotype d'une mutation germinale A366S devrait être une pseudohypoparathyroïdie "classique" cliniquement, mais remarquable biolo- giquement par le fait que l'activité de Gs mesurée in vitro, à température ambiante, ne serait pas diminuée, mais normale dans les conditions expérimentales utilisées habituellement pour le diagnostic de pseudohypoparathyroïdie. Celles-ci sont en effet différentes des conditions expérimentales utilisées par Bourne pour mettre en évidence l'activation de l'adénylyl cyclase par A366S : pour le 
diagnostic de pseudohypoparathyroïdie, l'incubation ne se fait pas en présence de GTP, mais d'un analogue non hydrolysable du GTP, le GTP $\gamma S$ [3]. Comme le GTP $\gamma S$ n'est pas hydrolysable, il suffit d'une très faible activité d'échange GDP/GTP pour déplacer l'équilibre vers la forme active $\alpha-G T P \gamma S$ et activer l'adénylyl cyclase. Ainsi, en présence de GTP $\gamma$ S, l'activation de l'adénylyl cyclase induite par la protéine mutante A366S est rigoureusement égale à celle induite par la protéine Gs normale, comme le montre d'ailleurs Bourne lui-même [1].

Or, cette activation de l'adénylyl cyclase mesurée en présence de GTP $\gamma \mathrm{S}$ est le critère utilisé pour séparer les deux formes de pseudohypoparathyroïdie de type 1: la pseudohypoparathyroïdie de type $1 \mathrm{~A}$, dans laquelle cette activité est diminuée de $50 \%$, et la pseudohypoparathyroïdie de type IB où elle est normale [7]. Il est intéressant de noter que la pseudohypoparathyroïdie de type lB, dont les mécanismes restent jusqu'à présent inconnus, recouvre en fait plusieurs phénotypes cliniques. Ce terme désigne, en effet, aussi bien des patients porteurs d'une résistance isolée à la PTH, que des patients, ou patientes, présentant une résistance hormonale multiple et une ostéodystrophie d'Albright, (c'est-à-dire le phénotype clinique de la pseudohypoparathyroïdie de type 1A) avec une "activité Gs normale». Au total, il est très probable que certaines patientes étiquetées "pseudohypoparathyroïdie de type 1B" avec ostéodystrophie, résistance hormonale multiple et "activité Gs normale» soient en fait porteuses de la mutation A366S.

Enfin, un autre phénotype pourrait être lié à l'acquisition somatique de la mutation A366S par une cellule de Leydig. Cet événement pourrait en effet suffire à provoquer le développement d'une tumeur testiculaire responsable d'hypersécrétion de testostérone. Il est donc possible que l'on retrouve prochainement une telle mutation dans des tumeurs testiculaires hypersécrétantes de l'enfant ou de l'adulte, ce qui élargira encore le champ des maladies endocriniennes liées à Gs

\section{RÉFÉRENCES}

1. Liri T, Herzmark P, Nakamoto JM, Van Dop C, Bourne HR. Rapid GDP release from $G s \alpha$ in patients with gain and loss of endocrine function. Nature $1994 ; 371: 1647$.

2. Clapham DE. Why testicules are cool. $\mathrm{Na}$ lure $1994 ; 371$ : 109-10.

3. Levine MA, Downs RW Ir, Moses AM, Breslau NA, Marx SJ, Lasker RD, Rizzoli RE, Aurbach GD, Spiegel AM. Resistance to multiple hormones in patients with pseudohypoparathyroidism. Association with deficient activity of guanine regulatory protein. Am J Med $1983 ; 74: 545-56$.

4. Patten JL, Johns DR, Valle D, Eil C, Gruppuso PA, Steele G, Smallwood PM, Levine MA. Mutations in the gene encoding the stimulatory $G$ protein of adenylate cyclase in Albright's hereditary ostetodystrophy. $N$ Engl 9 Med 1990 ; 322 : 1412-9.

5. Weinstein IS, Gejman PV, Friedman E, Kadowaki T, Collins RM, Gershon ES, Spiegel AM. Mutations of the Gs $\alpha$ subunit gene in Albright hereditary osteodystrophy detected by denaturing gradient gel electrophoted by den Natl Acad Sci USA $1990 ; 87: 8287-$
resis. Proc 90.

6. Shenker A Laue L, Kosugi S, Merendino Jr IJ, Mineghishi T, Cutler Ir GB. A constituvely activating mutation of the luteinizing hormone receptor in familial male precocious puberty. Nature $1993 ; 365: 652-5$

7. Mallet E. Hypo- et pseudo-hypoparathyroidies. Paris : Editions techniques, Encycl Med Chir, Pédiatrie, 4-106-A-10, 1994, 8 p.

\section{Olivier Chabre}

Service d'endocrinologie ( $\operatorname{Pr}$ Bachelot), CHU Albert Michallon,38043 Grenoble Cedex 9 et Inserm U. 244 (Pr Chambaz) DBMS, CENG, 17, avenue des Martyrs, 38054 Grenoble Cedex 9, France.

\section{TIRÉS À PART}

O. Chabre. 